\title{
Cooperative prediction for cognitive radio networks
}

\author{
S.D. Barnes • B.T. Maharaj • A.S. Alfa
}

\begin{abstract}
Combining spectrum sensing (SS) and primary user (PU) traffic forecasting provides a cognitive radio network (CRN) with a platform from which informed and proactive operational decisions can be made. The success of these decisions is largely dependent on prediction accuracy. Allowing secondary users (SU) to perform these predictions in a collaborative manner allows for an improvement in the accuracy of this process, since individual SUs may suffer from SS and prediction inaccuracies due to poor channel conditions. To overcome these problems a collaborative approach to forecasting PU traffic behaviour, that combines SS and forecasting through SU cooperation, has been proposed in this article. Both pre-fusion and post-fusion scenarios for cooperative prediction were investigated and a number of binary prediction methods were considered (including the authors' own simple technique). Cooperative prediction performance was investigated, under various PU traffic conditions, for a group of ten SUs experiencing different channel conditions and a sub-optimal cooperative forecasting algorithm was proposed to minimise cooperative prediction error. Simulation results indicated that the accuracy of the prediction methods was influenced by the PU traffic pattern and that cooperative prediction lead to a significant improvement in prediction accuracy under most of the traffic conditions considered. However, this came at the cost of increased computational complexity. The pre-fusion scenario was found to be the most accurate scenario (up to $25 \%$ improvement), but was also eleven
\end{abstract}

S.D. Barnes · B.T. Maharaj

Department of Electrical, Electronic and Computer Engineering, University of Pretoria, Pretoria, 0002, South Africa

Tel.: +27-11-9984403

E-mail: simonbarnes@ieee.org

A.S. Alfa

Department of Electrical and Computer Engineering, University of Manitoba, Canada 
times more complex than when no fusion was employed. The cooperative forecasting algorithm was found to further improve these results.

Keywords Cognitive Radio - Computational Complexity · Cooperative Prediction · Cooperative Sensing · Data Fusion · Occupancy Modelling

\section{Introduction}

A great deal has been published about spectrum scarcity in the literature of recent years. One of the technologies that addresses this problem is cognitive radio (CR) and CR networks (CRN) [20,16]. A critical function of a CRN is spectrum sensing (SS) and radio resource allocation [28]. A secondary user in a CRN will perform SS to gather information about the radio environment within which it wishes to operate and then use that information to make decisions about which channel it should immediately occupy. While SS on an individual basis is very useful to SUs it has been found that a cooperative approach, where SUs share their individual results, may provide SUs with more accurate information about the radio environment $[12,2]$.

It has also been shown in the literature that it is beneficial for SUs to be able to make proactive decisions about spectrum resource allocation $[17,25$, 8], both in terms of accuracy in channel selection and in the potential power savings for the entire CRN [11]. To be able to make these proactive decisions, a SU will need to be able to make predictions about the future behaviour of other users of the same spectrum. Various techniques have been presented in the literature that can be used to do this. However, it is important to consider the complexity that these techniques may introduce into the CRN and the possible negative side effects that this could lead to.

In this article the authors explore the premise that if collaboration between SUs may improve the SS process, then collaboration between SUs should also allow for better accuracy in the prediction process, needed for proactive decision making [10]. Firstly, a practical approach to implementing the maximum normal fit (MNF) algorithm for SS, a statistical approach to calculating the noise floor [5], is investigated. Secondly, the prediction accuracies of various prediction methods are compared under different traffic conditions. Thirdly, possible pre-fusion and post-fusion based prediction scenarios are introduced and their performance is compared to that of the single SU prediction scenario. Fourthly, a sub-optimal cooperative forecasting algorithm has been formulated to minimise the likelihood of cooperative prediction error and a heuristic for solving it proposed [9]. Finally, the issue of algorithmic complexity in the prediction process is also dealt with. An optimal balance is sought between the accuracy that forecasting and cooperation provides and the costs and delays that this may introduce into the CRN.

The rest of this article is organised as follows. Background information pertaining to the problem at hand is presented in Section 2. The system model that has been considered for the combination of cooperation and prediction is described in Section 3. Primary user detection is discussed in Section 4. 
Techniques for collaboratively modelling and forecasting PU behaviour are discussed in Section 5. Both the benefits and costs of cooperative prediction are then quantified via the simulation results presented and discussed in Section 7. Conclusions are then presented in Section 8.

\section{Background information}

\subsection{Traffic modelling}

Various approaches to modelling and predicting PU traffic have been proposed in the literature. One of these is the Markov chain (MC), which is a state based approach to the problem $[17,26,24,6]$. An alternative exponential model that assumes that PU arrival rate follows a Poisson process was presented in [14]. A linear regression model for predicting video traffic was proposed in [27] and methods based on adaptive filter theory have been described in $[1,19$, 18]. The concept of combining cooperation amongst SUs with prediction was briefly explored in [10], where it was suggested that a cooperative gain could be obtained if a large number of SUs with diverse channel characteristics could be employed.

However, while all of these methods aim to minimise prediction error, one of the problems associated with traffic prediction in a CRN is the computational complexity of the algorithm. This problem needs to be taken seriously, since PU traffic prediction needs to be performed quickly and accurately for it to be useful [8].

\subsection{Spectrum sensing}

CRs rely on a process, known as spectrum sensing (SS), to gather information about the radio environment in which they wish to operate [28]. This information allows them to make use of appropriate spectrum resources and also helps them to avoid interference with other uses of the spectrum. However, the accuracy of this information is of paramount importance since inaccurate data could negatively impact upon the performance of a CR network (CRN).

The probability of detection $P_{d, i}$ and the probability of error $P_{e, i}$ are measures that can be used to characterise SS performance. Consider a CRN scenario consisting of $N$ multiple secondary sensing nodes $S=\left[s_{1}, s_{2}, \ldots, s_{N}\right]$, where each node reports its individual decision to a centralised fusion centre (FC). Let the hypothesis that a channel is occupied be given by $H_{1}$ and that a channel is unoccupied by $H_{0}$. Also, let $E_{i}$ be the energy collected for sensing node $s_{i}$ and $\lambda_{i}$ be the threshold used by that sensor to determine channel occupancy. Then, using a technique known as energy detection, $P_{d, i}$ and $P_{e, i}$ may be defined as [28],

$$
\begin{aligned}
& P_{d, i}=\operatorname{Pr}\left\{E_{i}>\lambda_{i} \mid H_{1}\right\} \\
& P_{e, i}=P_{f a, i}+P_{m d, i}
\end{aligned}
$$


where $P_{m d, i}=1-P_{d, i}$ is the probability of mis-detection and $P_{f a, i}$ is the probability of false alarm, given as,

$$
P_{f a, i}=\operatorname{Pr}\left\{E_{i}>\lambda_{i} \mid H_{0}\right\} .
$$

A binary occupancy decision can be made using $E_{i}$ and $\lambda_{i}$, such that,

$$
D_{i}= \begin{cases}1, & E_{i}>\lambda_{i} \\ 0, & \text { otherwise }\end{cases}
$$

A statistical approach to calculating the noise floor, called the (MNF) method, may be employed to calculate $\lambda_{i}[5]$. The MNF method has been adopted to perform SS in this article and will be further investigated in Section 4 .

\subsection{Cooperative spectrum sensing}

One of the problems associated with SS is the hidden node problem [28], where due to severe shadowing or multipath fading, it is often not possible for a CR to obtain accurate results using only a single sensing node [22]. To counteract this issue, multiple sensing nodes can be employed to obtain a combined spectrum decision. Cooperative SS techniques thus often need to be employed so that accurate decisions can be made $[2,12]$. Since multiple cooperating sensing nodes are employed, the concept of a cooperative probability of detection $Q_{d}$ and a cooperative probability of false alarm $Q_{f}$ needs to be considered. Assuming the use of the $M$-out-of- $N$ fusion rule, these probabilities may be defined as [12],

$$
\begin{aligned}
& Q_{d}=\operatorname{Pr}\left\{\sum_{i=1}^{N} D_{i} \geq M \mid H_{1}\right\}, \\
& Q_{f}=\operatorname{Pr}\left\{\sum_{i=1}^{N} D_{i} \geq M \mid H_{0}\right\} .
\end{aligned}
$$

\section{Cooperative prediction scenario and system model}

\subsection{Prediction scenario}

A scenario, proposed by the authors, where multiple SUs cooperate to predict future spectrum availability is depicted in Fig. 1. It is assumed that all cooperating SUs are aware of the radio environment within which they are operating and that they are willing to collaboratively analyse the same channels. The aim of this collaboration is to generate a forecast for the future availability of the said band. The cooperative prediction scheme described in this article assumes the use of both a forecast engine (where predictions are made about future PU behaviour) as well as fusion centre (where information from cooperating 


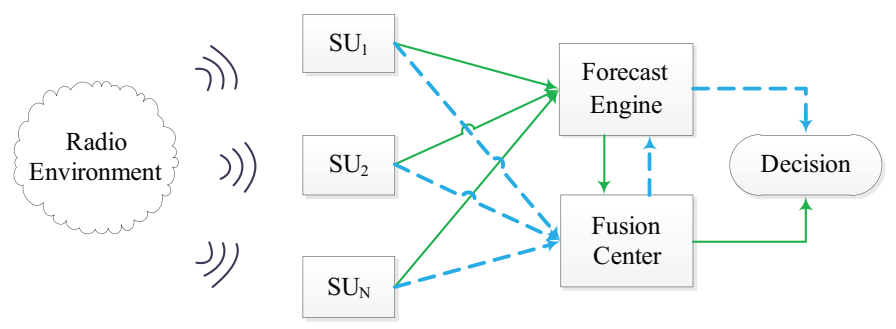

Fig. 1 Cooperative prediction scenarios

SUs is combined). Individual SUs perform SS, after which individual decisions are used to perform prediction based on fusing information. A decision is then made about the future availability of the band.

Two different cooperative fusion scenarios are illustrated in Fig. 1. Namely a pre-fusion scenario (illustrated by the solid green arrows) where each SU performs prediction before fusion is performed, and a post fusion scenario (indicated by the dashed blue arrows) where prediction is only performed after the SS results of each SU have already been combined. These fusion scenarios will be discussed in greater detail in Section 5 .

\subsection{Traffic classification}

The main purpose of traffic prediction in CRNs, is to provide SU's with information about PU behaviour before it actually happens. In order for a SU to be able to make these predictions, certain PU behavioural properties need to be ascertained. These behavioural properties may then be utilised in the prediction process, e.g., if PU behavioural patterns can be identified from historical data, then it is plausible that these patterns may be repeated in the future.

The first step in identifying these patterns is to classify the type of traffic that is to be predicted. In this article it is assumed that at time $t$, for a particular frequency band $\vartheta$, a SU $i$ will gather a sequence of binary occupancy decisions (ON and OFF periods) $\boldsymbol{S}_{t, i, \vartheta}(n)$ that describe the occupation of the band by a PU for a period of $p$ historical ON and OFF periods. This sequence is given as,

$$
\boldsymbol{S}_{i, t, \vartheta}(n)=\left\{D_{t-1, i}, D_{t-2, i}, \ldots, D_{t-p, i}\right\}
$$

An ON period denotes the presence of a PU (e.g. at $t=t-p, D_{t-p}=1$ ) and an OFF period the absence thereof (e.g. at $\left.t=t-p, D_{t-p}=0\right)$. This sequence is then used to model the broader traffic pattern generated by the PU.

The alternative exponential ON-OFF model has been adopted for modelling these ON and OFF periods [25]. For this model, it is assumed that channel occupancy can be modelled as an independently exponentially distributed processes, where the time intervals between the arrival and departure 
(a)

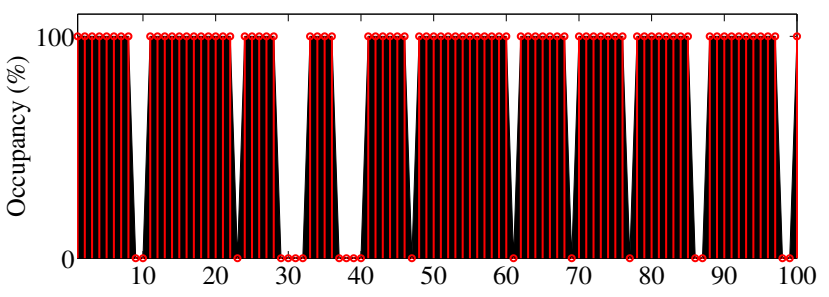

(b)

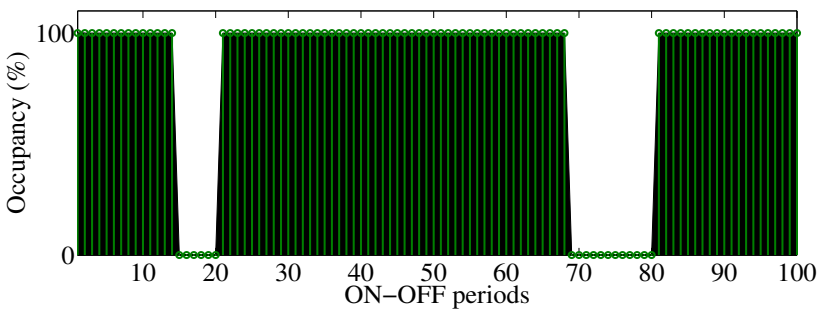

Fig. 2 Binary data sets illustrating: (a) fast, $\tau=1$, and (b) slow varying, $\tau=5$, PU activity (approximately $80 \%$ channel utilisation, $v_{0}=2$ and $v_{1}=8$ )

of a PU follow exponential distributions. For channel $\vartheta$ the OFF period distribution is given as,

$$
f\left(t_{\vartheta, 0}\right)= \begin{cases}\Lambda_{0} e^{-\Lambda_{0} t_{\vartheta, 0},} & t_{\vartheta, 0} \geq 0 \\ 0, & t_{\vartheta, 0}<0\end{cases}
$$

with mean $1 / \Lambda_{0}$ and $t_{\vartheta, 0}$ the duration of an OFF period. Similarly, the ON period distribution is given as,

$$
f\left(t_{\vartheta, 1}\right)= \begin{cases}\Lambda_{1} e^{-\Lambda_{1} t_{\vartheta, 1},}, & t_{\vartheta, 1} \geq 0 \\ 0, & t_{\vartheta, 1}<0\end{cases}
$$

with mean $1 / \Lambda_{1}$ and $t_{\vartheta, 1}$ the duration of an ON period.

In this article, for the sake of simplicity, PU traffic will be classified according to the following two parameters: pattern change rate and the average utilisation of the band. Traffic patterns can be described by using these parameters to adjust $\Lambda_{0}$ and $\Lambda_{1}$ according to the following expression,

$$
\begin{aligned}
& \Lambda_{0}=\tau v_{0}, \\
& \Lambda_{1}=\tau v_{1} .
\end{aligned}
$$

The rate at which PU occupancy patterns are changing is described by $\tau$, while $v_{0}$ and $v_{1}$ are a measure of $\mathrm{ON}$ and $\mathrm{OFF}$ period density respectively.

As an example consider the data sets presented in Fig. 2 where both data sets are a binary representation of a high PU traffic density scenario consisting of a hundred ON-OFF periods (approximately $80 \%$ channel utilisation) where $v_{0}=2$ and $v_{1}=8$. The first scenario (a), shown at the top of the figure, represents the case where $\mathrm{PU}$ activity is changing quite fast $(\tau=1)$. The 
second scenario (b), shown at the bottom of the figure, represents the case where PU activity is changing more slowly $(\tau=5)$.

The effect that $\tau$ and $v$ have on prediction performance is briefly investigated in Section 7.

\subsection{Radio environment}

Taking into account both large and small scale path loss, the received signal $r_{i}(n)$ at any $\mathrm{SU} i$ was assumed to be given by the following expression,

$$
r_{i}(n)=x(n) a_{i}(n) L_{i}+\gamma(n)
$$

where $x(n)$ is the transmitted PU signal, $a_{i}(n)$ is the fading on the channel between $\mathrm{SU} i$ and the PU base station (BS), $L_{i}$ is the free space path loss experienced by SU $i$ and $\gamma(n)$ is additive white Gaussian noise (AWGN). A frequency flat fading environment has been assumed, where the channel follows a Rician distribution,

$$
f_{i}(x)=\frac{x}{\sigma^{2}} \exp \left\{-\frac{x^{2}+\rho^{2}}{2 \sigma^{2}}\right\} I_{0}\left\{\frac{x \rho}{\sigma^{2}}\right\},
$$

with Rician $K$-factor,

$$
K=10 \log \left(\frac{\rho^{2}}{2 \sigma^{2}}\right)
$$

where $\rho$ is the line of site (LOS) amplitude, $I_{0}$ is the zero order modified Bessel function and $\sigma^{2}$ is the noise variance of the signal.

The geographical distribution of SUs, considered for this article, is illustrated in Fig. 3. The green line represents the cell boundary of a PU base station (BS) (shown as a red square), while ten cooperating SUs are illustrated as blue circles. The radius of the PU cell is $R=1.414 \mathrm{~km}$. Each SU, SU1 through SU10, has been assigned a different Rician $K$-factor ( $K=$ $[0 ; 18 ; 9 ; 1 ; 14.5 ; 4 ; 20 ; 6 ; 8 ; 12])$. The $K$-factor describes the severity of fading on the channel, which means that each $\mathrm{SU}$ will be able to detect PU activity with different degrees of success.

It is assumed that no errors are made when the SUs report either their individual SS or forecast results to the fusion centre (FC). Large scale fading effects such as diffraction and scattering have been ignored. The assumption has also been made that channel conditions change at a slower rate than the time for which a prediction has been made, i.e. the PU traffic pattern must not change during the predicted time slots. A necessary condition, to support this assumption, is that SUs remain stationary during this time (the geographical distribution of SUs must remain constant so that no Doppler shift can interfere with the sensing and prediction process). 


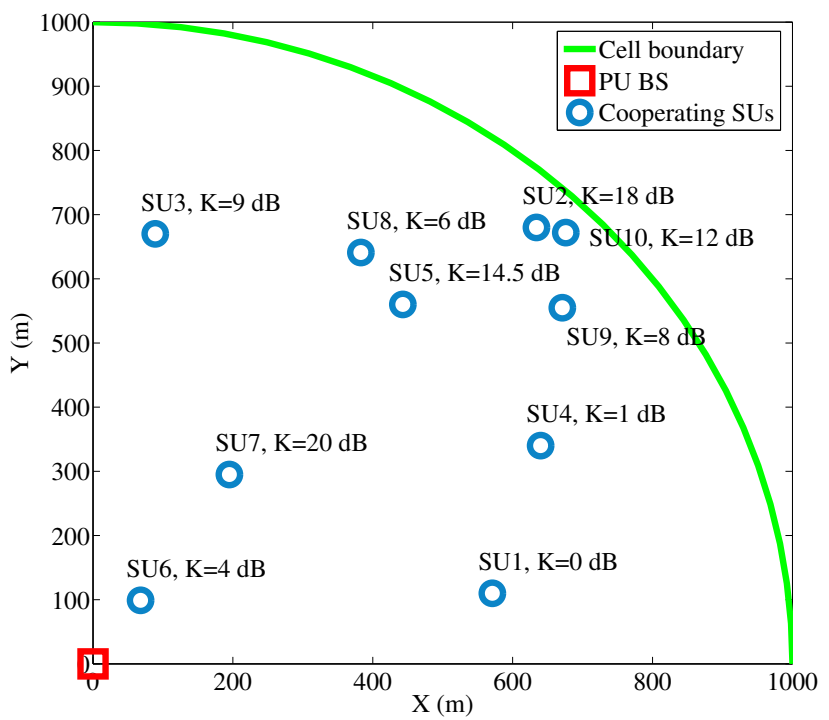

Fig. 3 Geographical distribution of cooperating SUs

\section{Primary user detection}

The procedure by which the SUs analyse and become aware of the channels within which they are operating is commonly referred to as SS. In this article a form of energy detection [23] was employed to detect the presence of PUs, where the noise energy threshold $\lambda$ was calculated using the MNF method [5]. This approach allows SUs to detect PU activity and calculate $\boldsymbol{S}_{i, t, \vartheta}(n)$ based on the value obtained for $r_{i}(n)$ in Eq. (11). This crucial process is the first step in the cooperative prediction process. Information gathered during this process is used to model historical PU activity as well as to verify prediction accuracy.

\subsection{Noise threshold calculation}

While the concept behind the MNF algorithm was discussed in our previous work [5], details were not clearly provided on how to practically implement it. A discussion now follows on how to calculate $\lambda$ using the MNF method given $r(n)$.

In the MNF method, the noise energy threshold is assumed to be the intersection of the noise and signal probability density functions (PDF) of Eq. (11). If both of these PDFs are assumed to follow a Gaussian distribution, where the noise distribution $\rho_{n}=\mathcal{N}\left(0, \sigma_{n}^{2}\right)$ is assumed to have zero mean and the signal distribution $\rho_{s}=\mathcal{N}\left(\mu_{s}, \sigma_{s}^{2}\right)$ some non-zero mean $\mu_{s}$, then the threshold $\lambda_{i}$, for $\mathrm{SU} i$, can be calculated from the following expression (derived 
in the appendix),

$$
\lambda_{i}=\frac{\mu_{s} \sigma_{n}^{2}-\sigma_{n} \sigma_{s} \sqrt{2 \ln \left(\frac{\sigma_{n}}{\sigma_{s}}\right)\left(\sigma_{n}^{2}-\sigma_{s}^{2}\right)+\mu_{s}^{2}}}{\sigma_{n}^{2}-\sigma_{s}^{2}} .
$$

If $r_{i}(n)$ represents the received signal of SU $i$, then the Gaussian assumption for $\rho_{s}$ requires that only the signal bearing portion of $r_{i}(n)$ be considered when calculating Eq. (14). The signal distribution $\rho_{s}$ is thus calculated as the PDF of $r_{i}(n)$ using only its positive elements. In other words, the signal portion $r_{i, s}(m)$ is roughly estimated to be,

$$
r_{i, s}(m)=r_{i}(n), \quad r_{i}(n) \geq 0
$$

and the PDF of the signal portion is then estimated by using $r_{i, s}(m)$, such that $\rho_{s}=\rho\left(r_{i, s}(m)\right)$. Therefore, the mean of the signal distribution can be calculated as,

$$
\mu_{s}=\frac{1}{M} \sum_{m=1}^{M} r_{i, s}(m)
$$

where $M \leq p$ is the number of positive elements in $r_{i}(n)$.

An estimate for the variance of the signal distribution $\sigma_{s}$ is obtained by firstly calculating the variance $\sigma_{r}^{2}$ and power $P_{r, i}$ of the received signal $r_{i}(n)$. The received power can be calculated as,

$$
P_{r, i}=\frac{1}{p} \sum_{n=1}^{p}\left|r_{i, s}(n)\right|^{2},
$$

and the variance as,

$$
\sigma_{r, i}=\frac{1}{M} \sum_{m=1}^{M}\left(r_{i}(n)-\mu_{s}\right)^{2}
$$

An estimate for the signal-to-noise ratio (SNR), denoted as $\gamma$, is then used, together with $P_{r, i}$, to obtain an estimate for the variance of the noise $\sigma_{n}^{2}$, such that,

$$
\sigma_{n}^{2}=\frac{P_{r, i}}{10^{(0.1 \gamma)}}
$$

The estimate for the variance of the signal component is then calculated as,

$$
\sigma_{s}^{2}=\sigma_{r, i}^{2}-\sigma_{n}^{2}
$$

Techniques that may be used to estimate $\gamma$ from received data include pilot assisted estimation [15] and various iterative minimum mean square error based techniques $[21,3]$. A perfect estimate for $\gamma$ was assumed in this article. 


\section{Cooperative prediction}

The historical behaviour of PUs can be used to model and then predict future behaviour, which can in turn be used by CRs to make proactive decisions. Multiple SUs may be used to collaboratively perform forecasting. In this article, this forecast is obtained by fusing the SS data obtained by multiple SUs to obtain a single improved forecast. In this section the manner in which prediction and fusion are combined will be investigated.

\subsection{Single and multi-user prediction}

In this article it will be assumed that, at one instant in time, prediction may be performed for either a single SU or concurrently for multiple SUs. In the single SU case, prediction is performed by a single SU, denoted by $i$. If a function $f(\cdot)$ is used to perform a forecast, then let $\boldsymbol{F}_{i, t, \vartheta}(r)$ be the sequence of predicted values for SU $i$ at time $t$ for frequency channel $\vartheta$, such that,

$$
\boldsymbol{F}_{i, t, \vartheta}(r)=f\left(\boldsymbol{S}_{i, t, \vartheta}(n)\right), \quad t+1 \leq r \leq t+k, \quad 1 \leq n \leq p
$$

where $k$ is the number of future samples predicted, $\{i, r, t, k\} \in \mathbb{N}, \boldsymbol{S}_{i, t, \vartheta} \in$ $\{0,1\}$ and $\boldsymbol{F}_{i, t, \vartheta}(r) \in\{0,1\}$.

For the multiple SU case a set, comprised of $N$ cooperating SUs, is used to calculate a combined forecast. Therefore, let the combined forecast, be denoted by $\boldsymbol{G}_{t, \vartheta}(r)$ such that,

$$
\boldsymbol{G}_{t, \vartheta}(r)=C^{N}\left\{\boldsymbol{F}_{i, t, \vartheta}(r)\right\}, \quad t+1 \leq r \leq t+k
$$

where $C^{N}\{\cdot\}$ indicates a combination of $N$ individual SU predictions.

\subsection{Prediction methods}

Five different prediction methods have been considered. These methods include: an approach based on MCs [13,24], the normalised least mean square (NLMS) approach [1,19], the first nearest neighbour approach (1-NN) [10] and a simple sliding occupancy window (OW) based method (proposed by the authors) [7]. These methods will all be compared to a uniformly distributed random prediction process.

The OW method is proposed as a simplified approach to prediction that relies on estimating the average utilisation of the band of interest over a window of observation of length $p$ time slots. Future predictions are then made as a hard decision, based on the calculated occupancy level of the observation window. The mean occupancy $\Phi$ of the observation window is calculated as follows,

$$
\Phi=\frac{1}{n} \sum_{j=1}^{n} \boldsymbol{S}_{i, t, \vartheta}(j) .
$$


Once $\Phi$ is known, the predicted sequence $\boldsymbol{F}_{i, t, \vartheta}(r)$ is calculated according to the following expression,

$$
\boldsymbol{F}_{i, t, \vartheta}(r)= \begin{cases}1, & \Phi \geq 0.5 \\ 0, & \Phi<0.5\end{cases}
$$

\subsection{Fusion rule}

When more than one SU begins to collaborate $(N>1)$, then the process of forecasting becomes a function of multiple SUs. There are a number of ways in which the information collected by individual SUs may be combined.

One of the most popular approaches to cooperative SS in the literature is the $M$-out-of- $N$ rule [4]. This is a voting based fusion rule which can be extended to cooperative forecasting. In this approach, a forecast value of $\boldsymbol{G}_{t, \vartheta}(r)=1$ is made if at least $M$ out of the $N$ cooperating SUs predict the presence of a $\mathrm{PU}$. The forecast is thus given as,

$$
\boldsymbol{G}_{t, \vartheta}(r)= \begin{cases}1, & \sum_{i=1}^{N} \boldsymbol{F}_{i, t, \vartheta}(r) \geq M \\ 0, & \text { otherwise }\end{cases}
$$

where $r=t+1, t+2, \ldots, t+k$.

The $M$-out-of- $N$ rule becomes the OR rule when $M=1$, and the AND rule when $M=N$. When using this approach, a suitable value for $M$ will need to be chosen. In this article, $M=\left\lceil\frac{N}{2}\right\rceil$ was used.

\subsection{Fusion scenarios}

Two fusion scenarios have been considered. In the first scenario, all collaborating SUs first perform prediction on a individual basis and then fuse their decisions (pre-fusion prediction). In the second scenario, collaborating SUs first fuse historical PU data at the fusion centre (post-fusion prediction) before performing a single prediction.

\subsubsection{Pre-fusion prediction}

The pre-fusion prediction scenario is illustrated by the block diagram shown in Fig. 4. Firstly, using Eq. (7), each collaborating SU obtains a sequence of SS results $\boldsymbol{S}_{i, t, \vartheta}(n)$, which is followed by an individual prediction by each SU about future channel availability $\boldsymbol{F}_{i, t, \vartheta}(r)$. After the prediction process, all of the individual SU predictions are fused together to obtained a combined prediction result $\boldsymbol{G}_{t, \vartheta}(r)$. The expressions given in Eq. (25) represent the prefusion prediction scenario. 


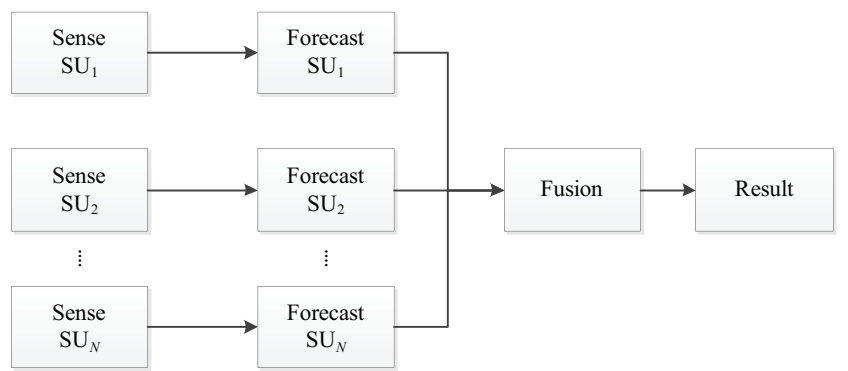

Fig. 4 Pre-fusion cooperative prediction

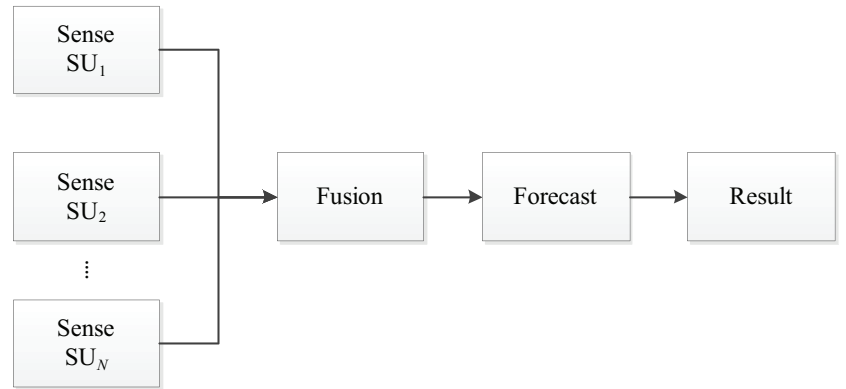

Fig. 5 Post-fusion cooperative prediction

\subsubsection{Post-fusion prediction}

The post-fusion prediction scenario is illustrated by the block diagram shown in Fig. 5. All collaborating SUs perform spectrum sensing, using Eq. (7), to obtain a sequence of historical PU information $\boldsymbol{S}_{i, t, \vartheta}(r)$. This data is then immediately fused to obtain a collaborative SS decision $\boldsymbol{H}_{t, \vartheta}(r)$, which may be described by the following expression,

$$
\boldsymbol{H}_{t, \vartheta}(n)= \begin{cases}1, & \frac{1}{N} \sum_{i=1}^{N} \boldsymbol{S}_{i, t, \vartheta}(n) \geq M \\ 0, & \text { otherwise }\end{cases}
$$

where $\{n, p\} \in \mathbb{N}$ and $\boldsymbol{H}_{t, \vartheta}(n) \in\{0,1\}$.

Only then is a single prediction made for future channel availability, using the result of the combined SS decision, such that,

$$
\boldsymbol{G}_{t, \vartheta}(r)=f\left(\boldsymbol{H}_{t, \vartheta}(n)\right), \quad t+1 \leq r \leq t+k, \quad 1 \leq n \leq p
$$

where $\{r, k\} \in \mathbb{N}$ and $\boldsymbol{G}_{t, \vartheta}(r) \in\{0,1\}$.

\subsection{Prediction approach}

The occupancy window prediction approach [7] was employed to calculate the local prediction error $\bar{\epsilon}_{f r}$. This error does not take into account the possibility 
that the information it uses to make the prediction may be incorrect due to poor SS, but is simply a prediction of future PU traffic based on information obtained through SS by a single SU. The following equation may be used to calculate $\bar{\epsilon}_{f r}$, the difference between the actual $\boldsymbol{X}_{t}(r)$ and predicted $\boldsymbol{F}_{t, \vartheta}(r)$ data sequences at time $t$,

$$
\bar{\epsilon}_{f r}=\frac{\sum_{r=1}^{k} \boldsymbol{F}_{t, \vartheta}(r)-\boldsymbol{X}_{t, \vartheta}(r)}{k}
$$

Each SU $i$ will detect different versions of $\boldsymbol{S}_{i, t, \vartheta}(n)$ due to imperfect SS and may also experience variations in its ability to preform a local forecast. To allow for variations in local prediction error $\bar{\epsilon}_{f r}$, the local forecast error for a single SU $i$ has been defined as,

$$
\epsilon_{f r, i, t}=v_{i, t} \bar{\epsilon}_{f r}
$$

where $v_{i, t}$ is randomly chosen from a continuous uniform distribution with probability density function $f_{v}(x)$ and a lower boundary of $\varsigma$, such that,

$$
v_{i, t}=\varsigma+(1-\varsigma) f_{v}(x) \text {. }
$$

For the simulation results shown in Section 7, the lower boundary $\varsigma$ acts as a spreading factor that allows for accuracy variations among cooperating SUs.

\section{Optimal forecasting}

The idea behind a cooperative prediction scheme is to minimise cooperative prediction error (CPE) by exploiting diversity amongst SUs. However, in order to so, it is necessary to perform predictions, and combine the data collected by cooperating SUs, in as optimal a fashion as possible. Therefore, an algorithm is presented in this section based on the system model and cooperative prediction approach described in Section 5 [9].

\subsection{Problem formulation}

Using Equation (27) the CPE at time $t$, denoted by $\epsilon_{c p, t}$, may be given as,

$$
\epsilon_{c p, t}=\frac{\sum_{j=1}^{k} \boldsymbol{G}_{t, \vartheta}(j)-\boldsymbol{X}_{t, \vartheta}(j)}{k}
$$

where $k$ is the number of future samples predicted at time $t$ on channel number $\vartheta$. Since it is desirable to minimise $\epsilon_{c p, t}$, the cooperative prediction problem 
may be formulated as,

$$
\begin{array}{cl}
\min _{\epsilon_{c p, t}} & \epsilon_{c p, t}=\frac{\sum_{i=1}^{k} \boldsymbol{G}_{t}(i)-\boldsymbol{X}_{t}(i)}{k}, \\
\text { s.t. } & 1 \leq n \leq p \\
& 1 \leq k \leq z \\
& 1 \leq i \leq N_{m x} \\
& 0 \leq \epsilon_{c p, t} \leq \bar{\epsilon}_{c p} \\
& n<t \leq \xi \\
& \rho, \nu \geq 1 \\
& 1 \leq \tau \leq \xi \\
& 0 \leq \varsigma \leq 1 \\
& p, k, n, z, i, t, \tau, \rho, \nu \in \mathbb{N} \\
& \boldsymbol{G}_{t, \vartheta}, \boldsymbol{X}_{t, \vartheta} \in\{0,1\} .
\end{array}
$$

In Equation (32), $n$ is the number of observations gathered by the local prediction algorithm and may not exceed the limit of $p$ observations, $z$ is the maximum number of future samples that may be predicted, $i$ is the number of cooperating SUs, $N_{m x}$ is the maximum number of cooperating SUs that may be combined, $\bar{\epsilon}_{c p}$ is the maximum error threshold for cooperative prediction, $\xi$ is the maximum number of iterations allowed by the algorithm (assuming a single iteration at each time increment), $\rho$ is the observation length resolution, $\nu$ is the amount by which $i$ may be changed, $\tau$ is the iteration interval that determines the sign of $\rho$ and $\varsigma$ controls the variation in prediction accuracy experienced by individual SUs (using Equation 29).

\subsection{Cooperative forecasting algorithm}

A cooperative forecasting algorithm (CFA) can be employed to find a solution for Equation (32). A sub-optimal heuristic for this problem is proposed and described in Algorithm 1. In this case, it is assumed that cooperating SUs report their individual forecasts to a centralised FC to calculate $G_{t, \vartheta}(n)$.

The CFA begins by calculating $F_{i, t, \vartheta}(r)$ for a single SU $(i=1)$ at time $t=0$, with $\{n, i, z\}=1$ initially. It then enters a recursive process that begins by calculating $G_{t, \vartheta}(n)$ for SU $i$ and the corresponding value for $\epsilon_{c p, t}$ $\left(G_{t, \vartheta}(r)=F_{i, t, \vartheta}(r)\right.$ at $\left.t=0\right)$. If $\epsilon_{c p, t}$ is found to be greater than the cooperative error threshold $\bar{\epsilon}_{c p}$ on the first iteration of the loop $(t=0)$, then the CFA will increase $i$ and $n$ by an amount $\rho$ and $\nu$ respectively $\rho, \nu \in \mathbb{N}$ (these variables represent the amount by which $i$ and $n$ will be adjusted at each iteration of the CFA).

This process is repeated for $\tau$ iterations. If after $\tau$ iterations $\epsilon_{c p, t}>\epsilon_{c p, t-\tau}$, then $n$ is decreased by $\rho$ and $i$ is kept constant to allow for the possibility that increasing $n$ may actually degrade prediction performance. This is repeated 


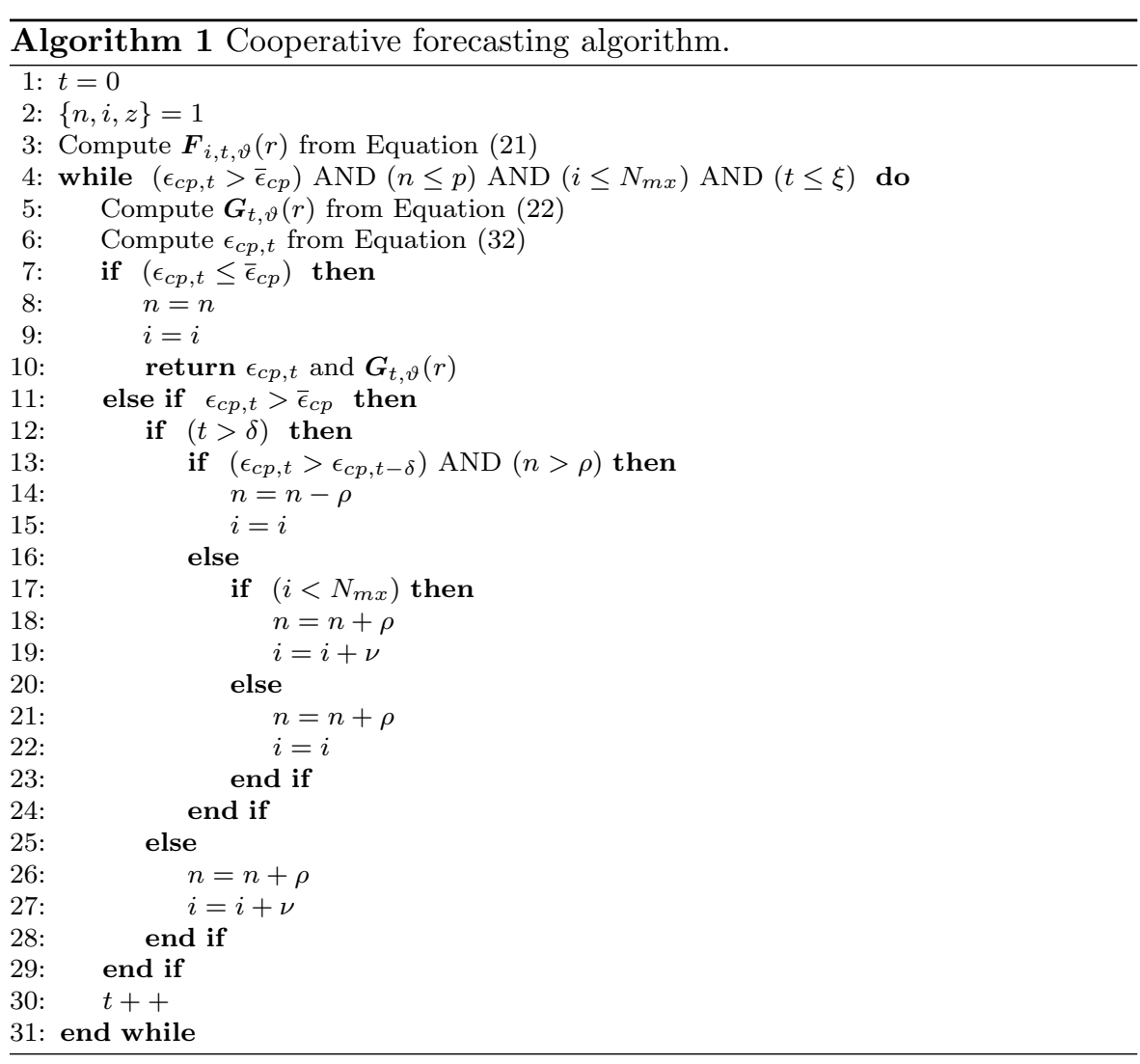

until either $\epsilon_{c p, t} \leq \epsilon_{c p, t-\tau}$ or $n \leq \rho$. Thereafter either both $i$ and $n$ are increased (when $i<N_{m x}$ ) or only $n$ is increased (if $i=N_{m x}$ ), at every iteration of the algorithm.

The CFA repeats itself until one of the stopping criteria are met: Either a lower cooperative prediction error than the threshold is found $\epsilon_{c p, t} \leq \bar{\epsilon}_{c p}$, the maximum number of historical observations has been exceeded $n>p$, the maximum number of cooperating SU has been exceeded $i>N_{m x}$ or the maximum number of iterations allowed by the algorithm has been reached $t>\xi$.

\section{Simulation results}

In this section, simulation results are presented to verify the performance of the suggested cooperative prediction techniques. Using MATLAB, three different simulations were performed, firstly to investigate SS, then single SU prediction and finally cooperative prediction. 
Table 1 Parameters for simulations one and three

\begin{tabular}{ll}
\hline Parameter & Value \\
\hline$T$ & 60000 time samples \\
$\vartheta$ & 100 \\
$N$ & 10 \\
$M$ & $\left|\frac{N}{2}\right|$ \\
$f_{0}(\mathrm{MHz})$ & 700 \\
$P_{T X}(\mathrm{~W})$ & 30 \\
$\mathrm{BW}(\mathrm{kHz})$ & 200 \\
$R(\mathrm{~m})$ & 1414 \\
$\gamma(\mathrm{dB})$ & {$[-4,30]$} \\
$P_{f r}$ & $0.6,0.7,0.8,0.9,1.0$ \\
$L_{i}(\mathrm{~dB})$ & $70.9,80.3,81.6,85.9,86.4$, \\
& $86.5,86.8,88.1,88.7,88.9$ \\
$K_{i}(\mathrm{~dB})$ & $0,18,9,1,14.5,4,20,6,8,12$ \\
\hline
\end{tabular}

\subsection{Simulation environment}

Simulation parameters for the first and third simulations (SS and cooperative prediction) are summarised in Table 1, while simulation parameters for the second simulation (single SU prediction) are summarised in Table 2.

The first simulation was run to investigate the performance of the PU detection scheme described in Section 4. SS results were obtained for ten different SU scenarios for an SNR range of $\gamma=[0,30] \mathrm{dB}$ in $2 \mathrm{~dB}$ increments.

The second simulation compared the prediction performance of the prediction methods listed in Section 5, namely the MC, NLMS, 1-NN, OW and uniform random prediction methods. These methods were compared under the PU traffic patterns described in Section 3. The observation length was fixed at $p=11$ and both forecast lengths of $k=1$ and $k=10$ were considered. Simulations were run for ten different channel occupancy values over the range $v_{0}=[10,0]$ and $v_{1}=[0,10]$.

The third simulation investigated the performance of cooperative prediction when compared to prediction by a single SU. The difference between the prediction performance of the pre-fusion and post-fusion prediction scenarios was compared over an SNR range of $\gamma=[-4,30]$ ( $8 \mathrm{~dB}$ increments), for five forecast probability values in the range $P_{f r}=[0.6,1.0]$.

All of the simulations were run for a length of $T=60000$ time samples. For the first and third simulations, the CRN environment consisted of $\vartheta=100$

Table 2 Simulation two specific parameters

\begin{tabular}{llllll}
\hline Parameter & $v_{0}$ & $v_{1}$ & $\tau$ & $k$ & $p$ \\
\cline { 2 - 6 } Value & {$[10,0]$} & {$[0,10]$} & $\{1 ; 5\}$ & $\{1 ; 10\}$ & 11 \\
\hline
\end{tabular}


channels spaced $200 \mathrm{kHz}$ apart, beginning at $f_{0}=700 \mathrm{MHz}$. The transmit power of the PU base station was set to $P_{T X}=30 \mathrm{~W}$. The $N=10$ SUs were randomly distributed within a quarter circle with radius $R=1414 \mathrm{~m}$. The calculated free space path loss $L_{i}$ and the assigned Rician $K$-factor $K_{i}$ for each SU are listed in Table 1 . For the fusion rule $M=\lceil N / 2\rceil=5$ was employed.

\subsection{Spectrum sensing performance}

Simulations were run to test the performance of the SS approach described in Section 4 . The individual noise thresholds $\lambda_{i}$ and the resulting probabilities of error $P_{e, i}$ were calculated for each of the SUs shown in Fig. 3. The calculated values for $\lambda_{i}$ (shown in $\mathrm{dBm}$ ) have been plotted on the left hand side of Fig. 6 , while the SS error probabilities $P_{e, i}$ have been plotted on the right hand side.

The calculated threshold values $\lambda_{i}$ exhibited an approximately inverse proportional relationship to SNR. However, this was not found to be true for SNR values below $\gamma=6 \mathrm{~dB}$ (since there is a greater overlap between the distributions of the information bearing and noise components of the signal, it becomes harder to calculate estimates for $\mu_{s}, \sigma_{s}^{2}$ and $\sigma_{n}^{2}$ when $\left.\gamma \leq 6 \mathrm{~dB}\right)$. As expected $P_{e, i}$ improves as both the SNR and Rician $K$-factor are increased, since it becomes easier to estimate $\lambda_{i}$.

The $P_{e, i}$ values represent the ability that each $\mathrm{SU}$ has to detect the presence of the PU based on the quality of the signal that it can detect and were used as the basis of the cooperative prediction simulations.
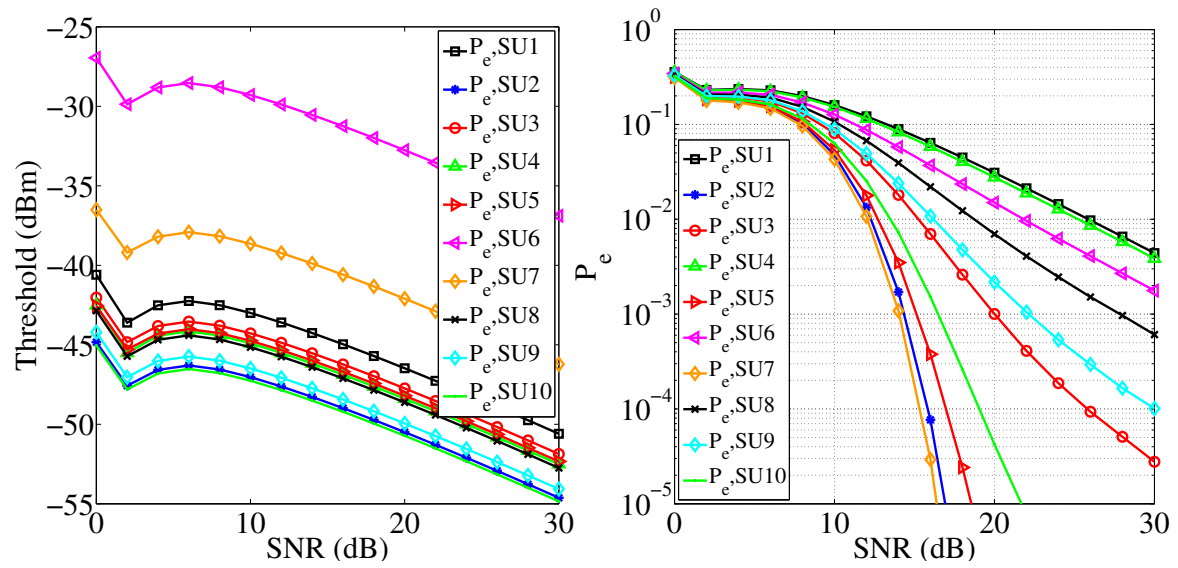

Fig. 6 Calculated detection thresholds (left) and SS error probabilities (right), as SNR is increased, for the SUs described in Section 3.3 
7.3 Single SU prediction performance

Simulations were also run to investigate the accuracy of the prediction methods discussed in Section 5. These prediction methods were tested using the data sets described in Section 3.2 and compared to the prediction accuracy that can be obtained by making random predictions according to a uniform distribution (the control method). The error incurred when making a prediction will henceforth be referred to as the prediction error (PE). Plots showing the $\mathrm{PE}$ over the full range of channel occupancy values are provided for fast changing PU traffic $(\tau=1)$ in Fig. 7 and for slow changing PU traffic $(\tau=5)$ in Fig. 8. For both traffic scenarios, an observation length of $p=11$ time samples was adopted and prediction lengths of $k=1$ and $k=10$ were considered.

It is evident that all of the prediction methods outperformed making random predictions (the control method) and that performance was better under slow changing traffic conditions. Also predictions further into the future (i.e. $k=10$ ) were found to be less accurate than for the immediate future (i.e $k=1)$. However, the most notable observation was that the $\mathrm{PE}$ of all of the methods was found to be the lowest when a band was either completely empty $(0 \%)$ or when it was completely occupied (100\%) and highest when the channel had an occupancy of $50 \%$. This can be explained by the effect of randomness on the prediction methods, since the PU traffic pattern is random when the occupancy is $50 \%$ and completely non-random when the occupancy is either $0 \%$ or $100 \%$. Similarly, a fast changing traffic pattern (e.g. $\tau=1$ ) can also make the PU traffic appear more random. Since the accuracy of the traffic prediction method relies on the existence of a repeatable PU traffic pattern, randomness will impair the prediction process. For a binary pattern, the
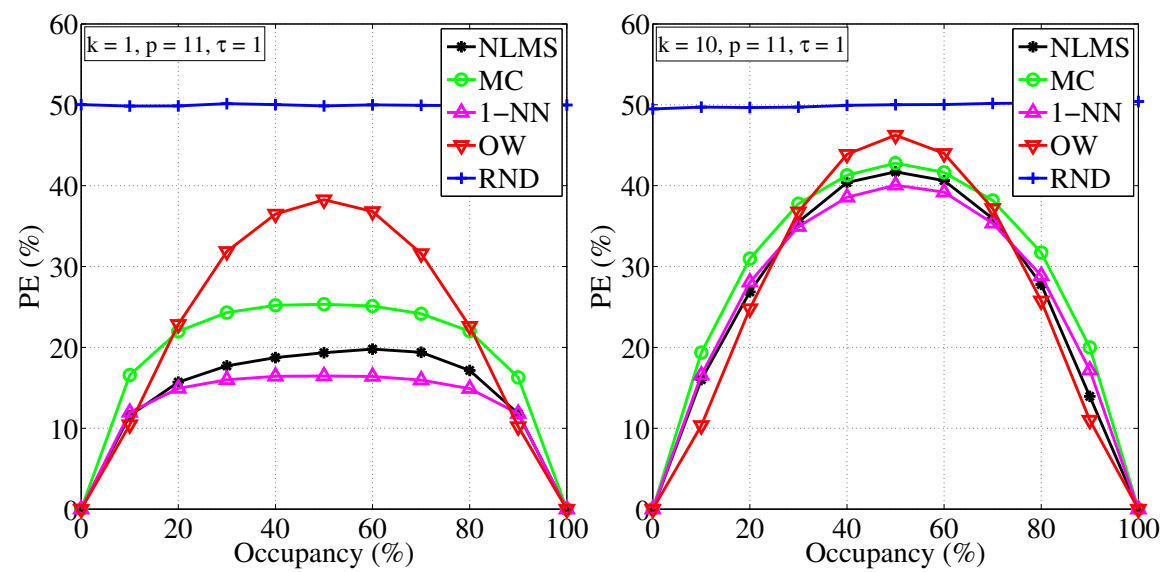

Fig. 7 Prediction error vs. channel occupancy for fast varying $\mathrm{PU}$ traffic $(p=11, k=$ $\{1,10\}, \tau=1)$ 
larger the number of contiguous bits that are the same, the easier it becomes to predict future bits.

There were individual differences between the performance of the prediction methods for different occupancy levels, traffic change rates and prediction lengths. These differences suggest that the choice of prediction method should be related to the traffic pattern of the actual channel for which a prediction is to be made. To highlight these differences, selected PE results are listed in Table 3.

Overall the 1-NN method (OW method when $p=1$ ) had the best performance, while the OW method was found to be the worst performing method. The OW method was particularly poor when the occupancy level was $50 \%$
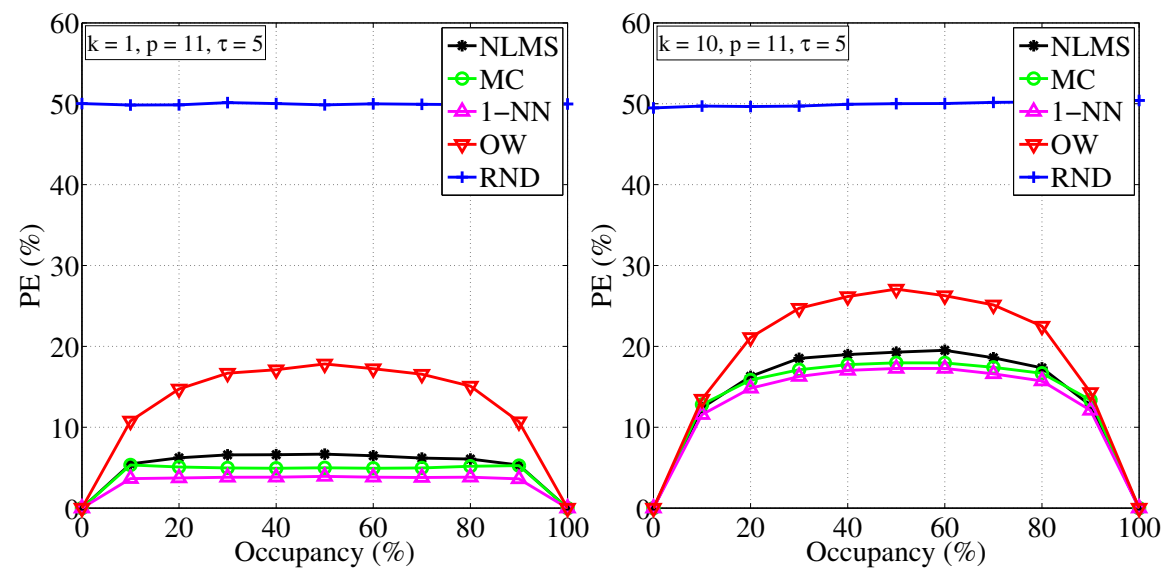

Fig. 8 Prediction error vs. channel occupancy for slow varying $\mathrm{PU}$ traffic $(p=11, k=$ $\{1,10\}, \tau=5)$

Table 3 Selected PE results for $v_{1}=5$ and $v_{1}=8$

\begin{tabular}{rccccc}
\hline \multirow{2}{*}{ Method } & \multicolumn{2}{c}{$\tau=1$} & & \multicolumn{2}{c}{$\tau=5$} \\
\cline { 2 - 3 } \cline { 5 - 6 } & $k=1$ & $k=10$ & & $k=1$ & $k=10$ \\
\hline$v_{1}=5$ & & & & \\
NLMS & 19.35 & 41.71 & & 6.67 & 19.29 \\
MC & 25.33 & 42.78 & & 4.99 & 17.97 \\
$1-N N$ & 16.46 & 40.06 & & 3.92 & 17.27 \\
OW & 38.26 & 46.24 & & 17.82 & 27.09 \\
RND & 49.87 & 50.02 & & 49.93 & 49.97 \\
$v_{1}=8$ & & & & \\
NLMS & 17.15 & 27.79 & & 6.07 & 17.37 \\
MC & 22.00 & 31.72 & & 5.18 & 16.68 \\
$1-N N$ & 14.91 & 28.87 & & 3.82 & 15.72 \\
OW & 22.61 & 25.75 & & 15.09 & 22.50 \\
RND & 49.89 & 50.23 & & 50.19 & 50.30 \\
\hline
\end{tabular}


$\left(v_{1}=5\right)$. However, the $\mathrm{PE}$ of all of the methods, became more similar as $v_{1}$ either approached zero or a hundred. Under certain conditions the OW method actually had the lowest PE. This occurred when predictions were made further into the future $(k=10)$ and the the traffic pattern was fast changing $(\tau=1)$.

The PEs of the MC and NLMS methods were found to be similar for slow changing traffic $(\tau=5)$, with the MC method marginally better. However, for a faster varying traffic pattern $(\tau=1)$, the NLMS method was found to be better, with a slightly larger difference between them (particularly when $k=1$ ).

While PE is of primary importance to the proactive decision making process, the complexity of each method could influence its viability in a CRN. This will be discussed in Section 7.6.

\subsection{Cooperative prediction performance}

To quantify the benefit that cooperation brings to the prediction process, simulations were run that compared the $\mathrm{CPE}$ that could be obtained by employing both pre-fusion prediction (PRE) and post-fusion prediction (POF) as compared to the no-fusion scenario (NOF). For the PRE and POF scenarios all ten SUs where employed in the prediction process, while for the NOF scenario prediction was performed using only SU1 (shown in Fig. 3). SU1 was used for the NOF scenario as it was the SU that was least able to detect the presence of the PU due to the poor channel conditions at its geographical location. The simulations were run for the range of SNR values listed in Table 1.

Each scenario was tested for a range of forecast probabilities $P_{f r}=[0.6,1.0]$. $P_{f r}$ is the probability that a correct forecast was made by SU $i$ and is the inverse of the PE, such that $P_{f r}=1-\mathrm{PE}$. Thus $P_{f r}=0.6$ represents a poor prediction with a $\mathrm{PE}$ of $40 \%$, while $P_{f r}=1.0$ represents a perfect prediction with a $\mathrm{PE}$ of $0 \%$.
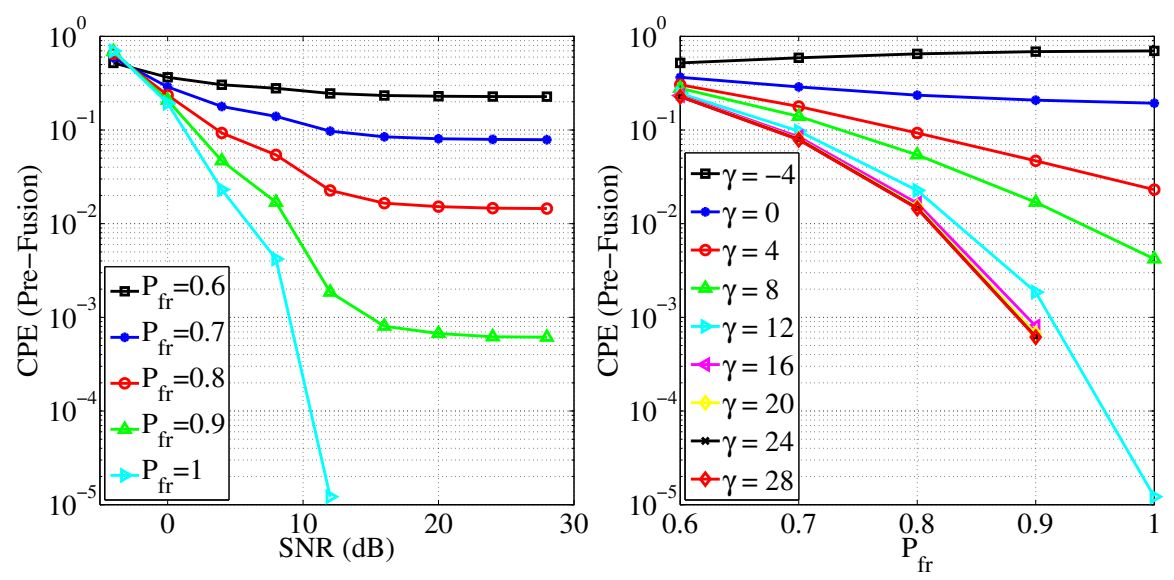

Fig. 9 Cooperative prediction error for the pre-fusion scenario 

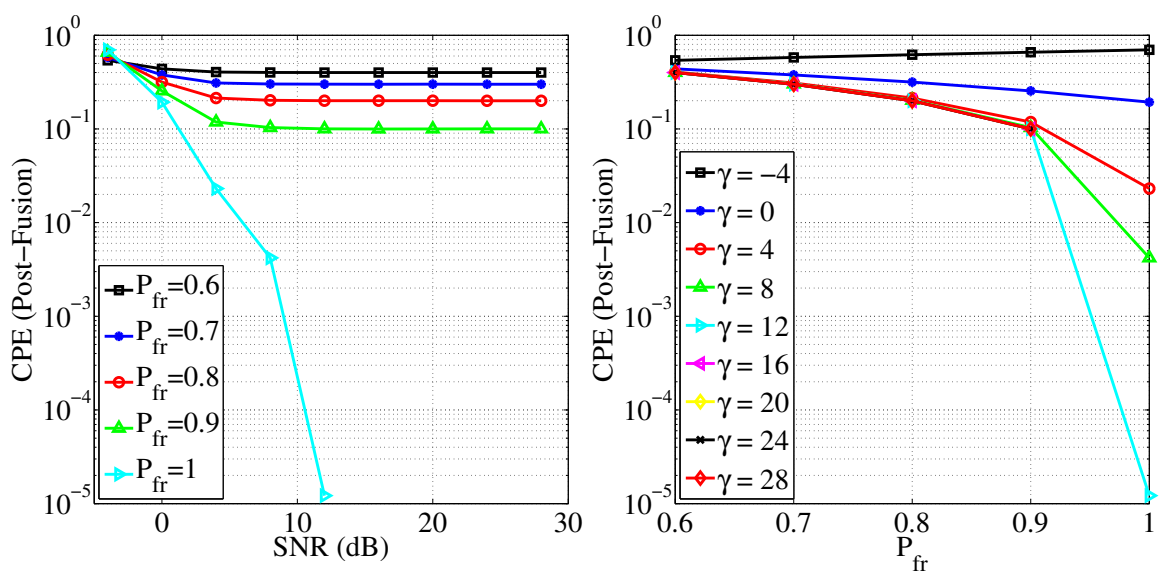

Fig. 10 Cooperative prediction error for the post-fusion scenario
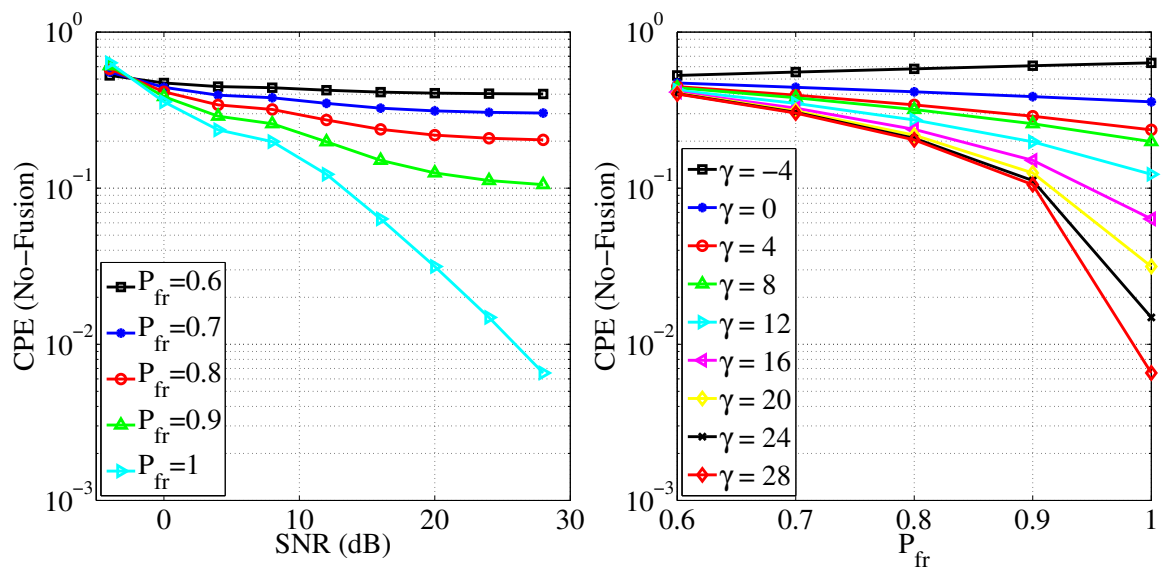

Fig. 11 Cooperative prediction error when fusion is not employed

The PRF results are illustrated in Fig. 9, the POF results in Fig. 10 and the NOF results in Fig. 11. For all three scenarios, the CPE improves exponentially as both the SNR and $P_{f r}$ are increased. However, this reduction in CPE is larger when fusion is employed for prediction, most notably when the PRF scenario is adopted. When fusion is employed the CPE can be reduced to a value that is below the $P_{f r}$ of the individuals SUs, as SNR is increased.

For the PRF scenario, the CPE when $P_{f r}<1.0$ continues to improve with an increase in SNR until it reaches its best value around $\gamma=24 \mathrm{~dB}$. For the POF scenario, however, the best $\mathrm{CPE}$ is reached much earlier at around $\gamma=8 \mathrm{~dB}$. This means that there is a benefit derived from fusion for a much wider range of noise conditions under the PRF scenario. Improved PE, due to cooperation, is evident at SNRs as low as $\gamma=0 \mathrm{~dB}$. 
Table 4 Selected PE results that highlight the benefit of cooperation

\begin{tabular}{rrrrrr}
\hline \multirow{2}{*}{ Scenario } & \multicolumn{2}{c}{$P_{f r}=0.7$} & & \multicolumn{2}{c}{$P_{f r}=0.9$} \\
\cline { 2 - 3 } \cline { 5 - 5 } & $\gamma=4$ & $\gamma=16$ & & $\gamma=4$ & $\gamma=16$ \\
\hline Single & & & & \\
SU1 & 39.43 & 32.55 & & 28.90 & 15.08 \\
Fusion & & & & \\
POF & 30.94 & 29.98 & & 11.84 & 9.99 \\
PRF & 17.81 & 8.46 & & 4.69 & 0.08 \\
Reduction & & & & \\
POF & 8.49 & 2.57 & & 17.06 & 5.09 \\
PRE & 21.62 & 24.09 & 24.21 & 15.00 \\
\hline
\end{tabular}

Selected results have been listed in Table 4 to highlight how much PE can be reduced by employing cooperative prediction. A CPE comparison is made for SNR values of $\gamma=4$ and $\gamma=16$, and for $P_{f r}=0.7$ and $P_{f r}=0.9$. When $\gamma=4 \mathrm{~dB}$, the POF scenario led to a CPE reduction of $8.49 \%$ and $17.06 \%$, for $P_{f r}=0.7$ and $P_{f r}=0.9$ respectively. However, the CPE was reduced by $21.62 \%$ and $24.21 \%$ respectively, for the PRE scenario. When $\gamma=16$, the difference between PRE and POF was even larger.

Thus it can be concluded that the PRF scenario provided much larger reductions in CPE than the POF scenario, while it was clear that both fusion scenarios are of benefit to the prediction process.

\subsection{Optimal cooperative prediction}

The CFA was used to calculate the results presented in Figure 12 and Figure 13.

\subsubsection{Uniform local prediction error}

In Figure 12, the CPE is illustrated for the case where $\varsigma=1$, i.e. for no diversity in local forecast error $\epsilon_{f r, i, t}$. Values for $\epsilon_{c p}$ have been plotted, on the left hand side of the figure, for each iteration of the CFA for various values of $\gamma$. The corresponding values for variables $i$ and $n$ are illustrated on the right hand side of the figure. From these plots it can been seen that $\epsilon_{c p}$ generally improved as the algorithm was iterated, reaching the optimal solution when $t=12$ after which no further improvement was made. This could be seen for all values of $\gamma$, where $i$ and $n$ steadily increased up to their optimal values, except for when $\gamma=0 \mathrm{~dB}$. When $\gamma=0 \mathrm{~dB}, i$ and $n$ sometimes had to be decreased to cope with local SS inaccuracies. This meant that it took longer to find the optimal solution, which was only reached when $t=16$. 


\subsubsection{Local prediction error diversity}

In Figure 13 the effect of diverse local prediction accuracy, when $\varsigma=[0,1]$, is illustrated. Using the CFA, the optimal value obtained for $\epsilon_{c p}$ is compared over a range of SNR values and for different values of $\varsigma$. Clearly diversity in the ability of SUs to perform local forecasts lead to a improvement in the CPE. For example, for $\gamma=16 \mathrm{~dB}$, the CPE obtained when $\varsigma=1.0$ was $\epsilon_{c p}=$ $6.405 \times 10^{-3}$, but due to diversity it had been reduced to $\epsilon_{c p}=3.800 \times 10^{-5}$ when $\varsigma=0.0$.

For lower SNR values there was an approximately exponential decrease in $\epsilon_{c p}$ with SNR, which then began to flatten out as SNR became larger. For $\varsigma=[0.4 ; 0.6 ; 0.8 ; 1.0]$, the minimum value for $\epsilon_{c p}$ appeared to have been reached
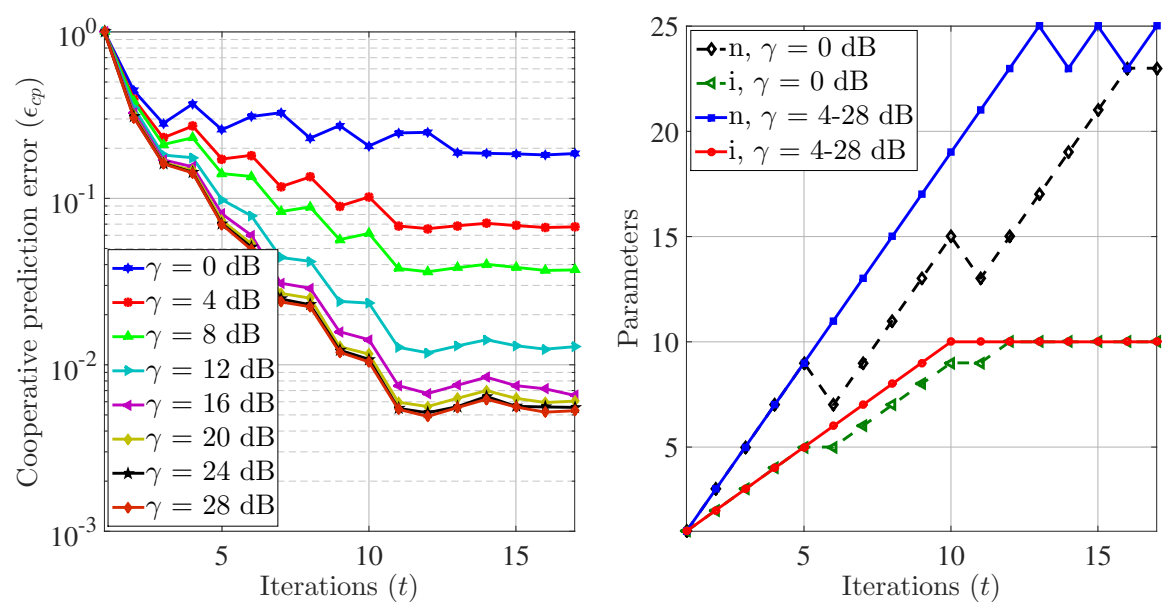

Fig. 12 Cooperative prediction error at each iteration of the CFA for different values of $\gamma$ (left) and the corresponding values for parameters $i$ and $n$ (right).

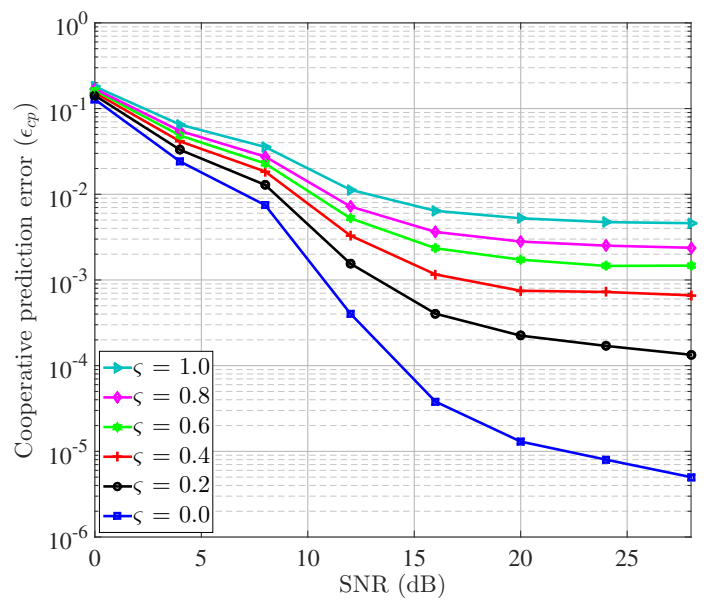

Fig. 13 Cooperative prediction error with local forecast diversity. 
(a)

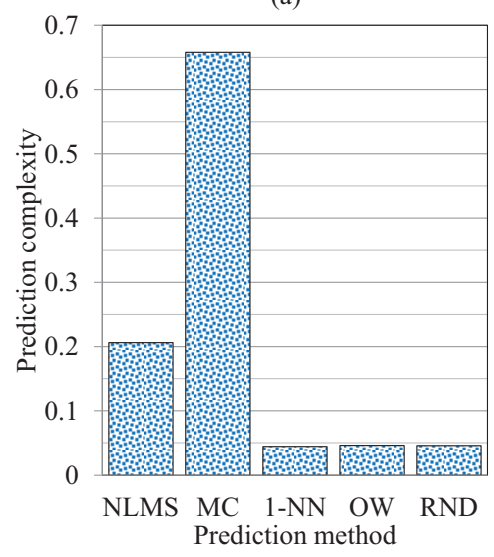

(b)

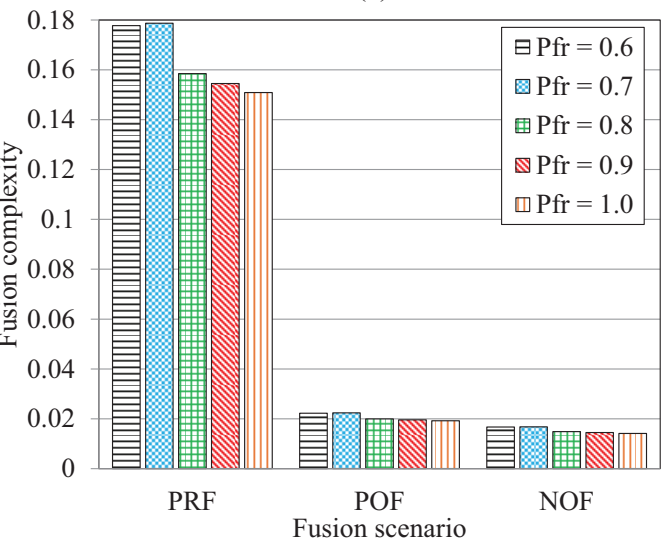

Fig. 14 Estimated complexity of (a) prediction method and (b) fusion scenario

when $\gamma=24 \mathrm{~dB}$. However, $\epsilon_{c p}$ was still decreasing at $\gamma=28 \mathrm{~dB}$ for a large diversity in local forecast accuracy $(\varsigma=[0.0 ; 0.2])$.

\subsection{The cost of cooperative forecast}

Although obtaining the lowest possible CPE is important, the complexity associated with cooperation and prediction must also be considered. Many CR functions are time critical and would be negatively affected if the SS and prediction process were to be an over complicated and time consuming process. Estimates for the complexities associated with each prediction method and fusion scenario, are illustrated in Fig 14. These estimates were calculated by normalising average simulation times using unit length feature scaling. The complexities of the prediction methods were calculated separately from the fusion scenarios.

The prediction method complexities are shown on the left hand side of Fig 14. Of the prediction methods that were simulated, the 1-NN method was found to be the least complex approach, closely followed by the OW method. Both of these methods were slightly less complex than the random approach, however, the MC and NLMS methods were found to be significantly more complex, with the MC method being 3.2 times more complex than the NLMS method and 14.5 times more complex than random prediction.

The fusion scenario complexities are shown on the right hand side of Fig 14 as well as in Table 5. As expected, there is a price to pay for the improvement in prediction accuracy due to fusion. For the PRF scenario, the most accurate approach, fusion was found to be 10.7 times more complex than for the NOF scenario (when $P_{f r}=0.8$ ). However, the POF scenario was found to be only 1.3 times more complex than the NOF scenario. 
Table 5 Selected complexity estimates for the prediction methods and fusion scenarios

\begin{tabular}{lrrrr}
\hline Scenario & $P_{f r}=0.6$ & $P_{f r}=0.7$ & $P_{f r}=0.8$ & $P_{f r}=0.9$ \\
\hline PRF & 0.178 & 0.179 & 0.158 & 0.154 \\
POF & 0.022 & 0.022 & 0.020 & 0.020 \\
NOF & 0.017 & 0.017 & 0.015 & 0.014 \\
\hline
\end{tabular}

Therefore, when performing cooperative prediction it is important to select the correct combination of prediction method and fusion scenario, so as to ensure a reasonable balance between prediction accuracy and complexity.

\section{Conclusion}

Accurate prediction of PU behaviour allows for proactive decision making in a CRN and collaboration amongst multiple SUs, during the prediction process, may lead to improved prediction accuracy. By cooperating with each other, SUs are potentially able to predict PU behaviour with greater accuracy than they would have on their own. In this article the performance of various prediction methods and fusion scenarios, for cooperative prediction, were investigated for different PU traffic conditions. A sub-optimal cooperative forecasting algorithm was also presented. From the simulation results obtained, cooperative prediction was shown to provide greater accuracy than when a single SU, experiencing poor channel conditions, was used to predict PU activity. The performance of the prediction methods was also found to be influenced by the density of PU traffic and the rate at which it was changing. The pre-fusion fusion scenario was clearly found to provide greater accuracy than the post fusion scenario, however, this was achieved at a significant increase in required computational complexity. A trade-off was thus found to exist between cooperative prediction accuracy and computational complexity. The cooperative forecasting algorithm was also found to be effective at reducing CPE even further. The findings of this work may provide a platform for future research relating to optimal PU traffic prediction.

Acknowledgements This research was supported by the Sentech Chair in Broadband Wireless Multimedia Communication (BWMC), the National Research Foundation (NRF) and the Independent Communications Authority of South Africa (ICASA).

\section{References}

1. Adas, A.M.: Using adaptive linear prediction to support real-time VBR video under RCBR network service model. IEEE/ACM Trans. Netw. 6(5), 635-644 (1998)

2. Akyildiz, I.F., Lo, B.F., Balakrishnan, R.: Cooperative spectrum sensing in cognitive radio networks: A survey. Physical Commun. 4(1), 40-62 (2011)

3. Arshad, A., Hassan, S.A.: Maximum likelihood SNR estimation for non-coherent FSKbased cooperative networks over correlated Rayleigh fading channels. In: Proc. IEEE Wireless Commun. Netw. Conf., pp. 636-641. New Orleans, United States (2015) 
4. Atapattu, S., Tellambura, C., Jiang, H.: Energy detection based cooperative spectrum sensing in cognitive radio networks. IEEE Trans. Wireless Commun. 10(4), 1232-1241 (2011)

5. Barnes, S.D., Jansen van Vuuren, P.A., Maharaj, B.T.: Spectrum occupancy investigation: Measurements in South Africa. Measurement 46(9), 3098-3112 (2013)

6. Barnes, S.D., Maharaj, B.T.: Performance of a hidden Markov channel occupancy model for cognitive radio. In: Proc. IEEE AFRICON Conf., pp. 1-6. Livingstone, Zambia (2011)

7. Barnes, S.D., Maharaj, B.T.: An occupancy window approach to primary user traffic modelling for cognitive radio. In: Proc. SATNAC, pp. 395-399. Stellenbosch, South Africa (2013)

8. Barnes, S.D., Maharaj, B.T.: Prediction based channel allocation performance for cognitive radio. AEU - International Journal of Electronics and Communications 68(4), 336-345 (2014)

9. Barnes, S.D., Maharaj, B.T.: Collaborative spectral opportunity forecasting for cognitive radio. In: Proc. IEEE AFRICON Conf., pp. 1-6. Addis Ababa, Ethiopia (2015)

10. Chen, Z., Guo, N., Hu, Z., Qiu, R.C.: Experimental validation of channel state prediction considering delays in practical cognitive radio. IEEE Trans. Veh. Technol. 60(4), 13141325 (2011)

11. Deng, R., Chen, J., Yuen, C., Cheng, P., Sun, Y.: Energy-efficient cooperative spectrum sensing by optimal scheduling in sensor-aided cognitive radio networks. IEEE Trans. Veh. Technol. 61(12), 716-725 (2012)

12. Ghasemi, A., Sousa, E.: Opportunistic spectrum access in fading channels through collaborative sensing. J. Commun. 2(2), 71-82 (2007)

13. Ghosh, C., Cordeiro, C., Agrawal, D.P., Rao, M.B.: Markov chain existence and hidden markov models in spectrum sensing. In: Proc. 7th Annu. IEEE Int. Conf. Pervasive Comput. Commun., pp. 1-6. Galveston, TX (2009)

14. Ghosh, C., Pagadarai, S., Agrawal, D.P., Wyglinski, A.M.: A framework for Statistical Wireless Spectrum Occupancy Modeling. IEEE Trans. Wireless Commun. 9(1), 38-44 (2010)

15. Hassan, S.A., Ingram, M.A.: Pilot assisted SNR estimation in a non-coherent M-FSK receiver with a carrier frequency offset. In: Proc. IEEE Int. Conf. Commun., pp. 36983702. Ottawa, Canada (2012)

16. Haykin, S.: Cognitive radio: brain-empowered wireless communications. IEEE J. Sel. Areas Commun. 23(2), 201-220 (2005)

17. Höyhtyä, M., Pollin, S., Mämmelä, A.: Performance improvement with predictive channel selection for cognitive radios. In: Proc. 1st Int. Workshop Cognitive Radio Adv. Spectr. Manage., pp. 1-5. Aalborg, Denmark (2008)

18. Kulkarni, P., Lewis, T., Fan, Z.: Simple traffic prediction mechanism and its applications in wireless networks. Wireless Pers. Commun. 59(2), 261-274 (2011)

19. Kuo, W.K., Lien, S.Y.: Dynamic resource allocation for supporting real-time multimedia applications in IEEE 802.15.3 WPANs. IET Commun. 3(1), 1-9 (2009)

20. Mitola III, J., Maguire Jr., G.Q.: Cognitive radio: making software radios more personal. IEEE Pers. Commun. 6(4), 13-18 (1999)

21. Savaux, V., Louët, Y., Djoko-Kouam, M., Skrzypczak, A.: Application of a joint and iterative MMSE-based estimation of SNR and frequency-selective channel for OFDM systems. Eurasip J. Advances Signal Process. 1(128), 1-11 (2013)

22. Unnikrishnan, J., Veeravalli, V.: Cooperative sensing for primary detection in cognitive radio. IEEE J. Sel. Topics Signal Process. 2(1), 18-27 (2008)

23. Urkowitz, H.: Energy detection of unknown deterministic signals. Proceedings of the IEEE 55(4), 523-531 (1967)

24. Wen, Z., Fan, C., Zhang, X., Wu, Y., Zou, J., Liu, J.: A learning spectrum hole prediction model for cognitive radio systems. In: in Proc. 10th IEEE Int. Conf. Comput. Inf. Technol., pp. 2089-2093. Bradford, United Kingdom (2010)

25. Yang, C.S., Chuang, L.Y., Chen, Y.J., Yang, C.H.: Feature selection using memetic algorithms. In: Proc. Int. Conf. Convergence Hybrid Inf. Technol., pp. 416-423. Busan, South Korea (2008) 
26. Yao, Y., Feng, Z., Li, W., Qian, Y.: Dynamic spectrum access with QoS guarantee for wireless networks: a Markov approach. In: Proc. IEEE Global Telecommun. Conf., pp. 1-5. Miami, FL (2010)

27. Yarkan, S., Arslan, H.: Binary time series approach to spectrum prediction for cognitive radio. In: Proc. IEEE Veh. Technol. Conf., pp. 1563-1567. Baltimore, MD (2007)

28. Yücek, T., Arslan, H.: A survey of spectrum sensing algorithms for cognitive radio applications. IEEE Commun. Surveys Tuts. 11(1), 116-130 (2009)

\section{Author Biographies}

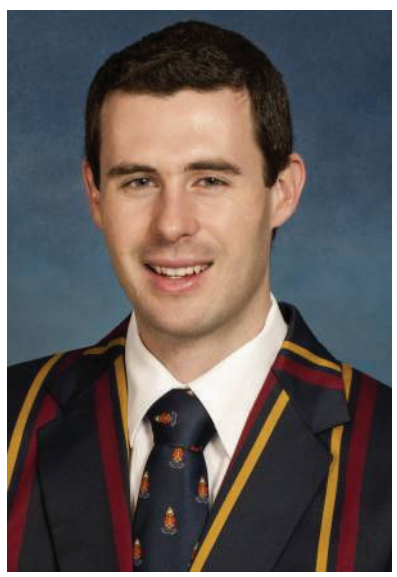

Simon Barnes received his M.Eng in Electronic Engineering, with distinction, from the University of Pretoria. He is a Ph.D candidate at the Sentech Chair in Broadband Wireless Multimedia Communications in the Department of Electrical, Electronic and Computer Engineering at the University of Pretoria. He is currently working as a senior electronic engineer at Tracker Connect (Pty) Ltd, Centurion, South Africa. His research interests include cognitive radio, dynamic spectrum access, software defined radio and spectrum utilisation.

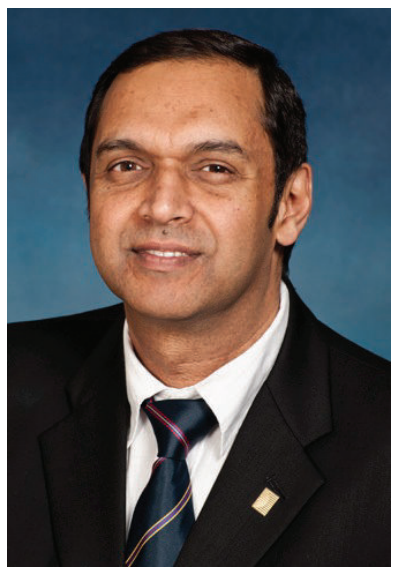

Sunil Maharaj received his Ph.D. in Wireless Communications from the University of Pretoria. Professor Maharaj currently holds the position of Sentechis the current Dean of the Faculty of Engineering, Built Environment and Information Technology at the University of Pretoria and the SENTECH Chair in Broadband Wireless Multimedia Communications in the Department of Electrical, Electronic and Computer Engineering at the same institution. His research interests are in OFDMMIMO systems, massive MIMO and cognitive radio resource allocation. 


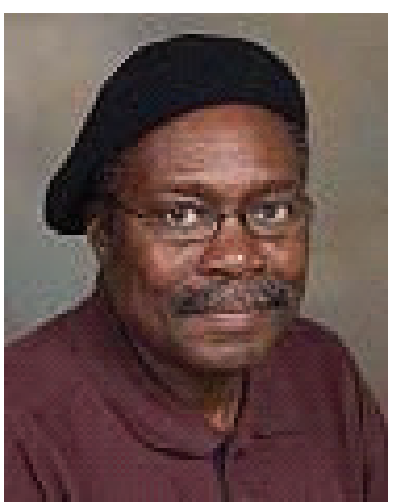

Attahiru Alfa Dr. Alfa is a Professor of telecommunication systems at the University of Manitoba, Department of Electrical and Computer Engineering. His research covers, but not limited to, the following areas: performance analysis and resource allocation in telecommunication systems, modelling of communication networks, queuing theory, optimization, analysis of cognitive radio networks, modelling and analysis of wireless sensor networks, developing efficient decoding algorithms for LDPC codes, channel modelling, traffic estimation for the Internet, and cross layer analysis.

\section{Appendix}

In this appendix the expression for $\lambda_{i}$ is derived.

Lemma 1 The signal detection threshold $\lambda_{i}$ is given by the following expression,

$$
\lambda_{i}=\frac{\mu_{s} \sigma_{n}^{2}-\sigma_{n} \sigma_{s} \sqrt{2 \ln \left(\frac{\sigma_{n}}{\sigma_{s}}\right)\left(\sigma_{n}^{2}-\sigma_{s}^{2}\right)+\mu_{s}^{2}}}{\sigma_{n}^{2}-\sigma_{s}^{2}}
$$

where $\mu_{s}$ and $\sigma_{s}^{2}$ are the mean and variance of the information carrying component of a received signal respectively and $\sigma_{n}^{2}$ is the variance of the noise component.

Proof Let the probability density functions of the information and noise components be,

$$
\rho_{n}(x)=\frac{1}{\sigma_{n} \sqrt{2 \pi}} \exp \frac{\left(x-\mu_{n}\right)^{2}}{2 \sigma_{n}^{2}}
$$

and

$$
\rho_{s}(x)=\frac{1}{\sigma_{s} \sqrt{2 \pi}} \exp \frac{\left(x-\mu_{s}\right)^{2}}{2 \sigma_{s}^{2}}
$$

respectively.

To solve for $x$, let $\rho_{n}(x)=\rho_{s}(x)$ such that,

$$
\frac{1}{\sigma_{n} \sqrt{2 \pi}} \exp \frac{\left(x-\mu_{n}\right)^{2}}{2 \sigma_{n}^{2}}=\frac{1}{\sigma_{s} \sqrt{2 \pi}} \exp \frac{\left(x-\mu_{s}\right)^{2}}{2 \sigma_{s}^{2}}
$$

This expression can be simplified and written in the form of a second order polynomial, $a x^{2}+b x+c=0$, where the coefficient are given as,

$$
\begin{aligned}
& a=x^{2}\left(\sigma_{n}^{2}-\sigma_{s}^{2}\right) \\
& b=2 x\left(\sigma_{s}^{2} \mu_{n}-\sigma_{n}^{2} \mu_{s}\right) \\
& c=\sigma_{n}^{2} \mu_{s}^{2}-\sigma_{s}^{2} \mu_{n}^{2}-2 \sigma_{n}^{2} \sigma_{s}^{2} \ln \left(\frac{\sigma_{n}}{\sigma_{s}}\right)
\end{aligned}
$$


If $\lambda_{i}=x$, is the solution for Eq. (36), then,

$$
\lambda_{i}=\frac{\mu_{s} \sigma_{n}^{2}-\mu_{n} \sigma_{s}^{2}}{\sigma_{n}^{2}-\sigma_{s}^{2}} \pm \frac{\sigma_{n} \sigma_{s} \sqrt{2 \ln \left(\frac{\sigma_{n}}{\sigma_{s}}\right)\left(\sigma_{n}^{2}-\sigma_{s}^{2}\right)+\mu_{n}^{2}+\mu_{s}^{2}-2 \mu_{n} \mu_{s}}}{\sigma_{n}^{2}-\sigma_{s}^{2}}
$$

But, under the assumption that $\rho_{n}=\mathcal{N}\left(0, \sigma_{n}^{2}\right), \mu_{n}=0$ must be substituted into Eq. (40) to give the following expression,

$$
\lambda_{i}=\frac{\mu_{s} \sigma_{n}^{2} \pm \sigma_{n} \sigma_{s} \sqrt{2 \ln \left(\frac{\sigma_{n}}{\sigma_{s}}\right)\left(\sigma_{n}^{2}-\sigma_{s}^{2}\right)+\mu_{s}^{2}}}{\sigma_{n}^{2}-\sigma_{s}^{2}}
$$

If it is assumed that $\mu_{s}>0$, then it follows that,

$$
\mu_{s} \sigma_{n}^{2}>\sqrt{2 \ln \left(\frac{\sigma_{n}}{\sigma_{s}}\right)\left(\sigma_{n}^{2}-\sigma_{s}^{2}\right)+\mu_{s}^{2}}
$$

Therefore, since $\lambda_{i} \leq \mu_{s}$, Eq. (41) becomes,

$$
\lambda_{i}=\frac{\mu_{s} \sigma_{n}^{2}-\sigma_{n} \sigma_{s} \sqrt{2 \ln \left(\frac{\sigma_{n}}{\sigma_{s}}\right)\left(\sigma_{n}^{2}-\sigma_{s}^{2}\right)+\mu_{s}^{2}}}{\sigma_{n}^{2}-\sigma_{s}^{2}}
$$

